\title{
When should reverse total shoulder arthroplasty be considered in glenohumeral joint arthritis?
}

\author{
Young-Hoon Jo ${ }^{1}$, Dong-Hong Kim ${ }^{2}$, Bong Gun Lee ${ }^{2}$ \\ ${ }^{1}$ Department of Orthopedic Surgery, Hanyang University Guri Hospital, Guri, Korea \\ ${ }^{2}$ Department of Orthopedic Surgery, Hanyang University College of Medicine, Seoul, Korea
}

\begin{abstract}
Anatomical total shoulder arthroplasty (TSA) has been used widely in treatment of glenohumeral osteoarthritis and provides excellent pain relief and functional results. Reverse total shoulder arthroplasty (RSA) was created to treat the complex problem of rotator cuff tear arthropathy. RSA also has been performed for glenohumeral osteoarthritis even in cases where the rotator cuff is preserved and has shown good results comparable with TSA. The indications for RSA are expanding to include tumors of the proximal humerus, revision of hemiarthroplasty to RSA, and revision of failed TSA to RSA. The purposes of this article were to describe comprehensively the conditions under which RSA should be considered in glenohumeral osteoarthritis, to explain its theoretical background, and to review the literature.
\end{abstract}

Keywords: Shoulder; Arthroplasty; Osteoarthritis; Rotator cuff; Stiffness

\section{INTRODUCTION}

Anatomical total shoulder arthroplasty (TSA) has been used widely in treatment of glenohumeral osteoarthritis (GHOA) and provides excellent pain relief and functional results [1-3]. As TSA is designed for restoring the biomechanics of a normal shoulder joint, adequate glenoid bone stock and intact rotator cuff tendons are essential for good results. Biomechanically, the TSA needs soft tissue balance and must permit translation in the glenohumeral joint. Reverse total shoulder arthroplasty (RSA) was created to treat the complex problem of rotator cuff tear arthropathy [4]. Biomechanically, the RSA provides a stable and fixed fulcrum of the arm for rotation, while increasing the moment arm and resting tension of the deltoid muscle, which enable arm ele- vation and abduction, even in massive rotator cuff tears [5,6]. For the last three decades, RSA for cuff tear arthropathy has been successful. RSA can be used not only for patients with cuff tear arthropathy, but also for those with other complex shoulder problems in whom the soft tissues or glenoid bone stock can be deficient. The indications for its use are expanding to include tumors of the proximal humerus, revision of hemiarthroplasty to RSA, and revision of failed TSA to RSA [4,6-8].

TSA requires restoration of the normal shoulder construct in soft tissue balance and bony architecture. If preoperative factors related with poor clinical outcomes in TSA are uncorrectable, satisfactory results cannot be obtained. In this situation, RSA could be an alternative option. Upon literature review, three factors have been mentioned commonly as related with poor clinical

Received: November 1, $2021 \quad$ Accepted: November 9, 2021

Correspondence to: Bong Gun Lee

Department of Orthopedic Surgery, Hanyang University College of Medicine, 222-1 Wangsimni-ro, Seongdong-gu, Seoul 04763, Korea

Tel: +82-2-2290-8485, Fax: +82-2-2299-3774, E-mail: orthdr@naver.com, ORCID: https://orcid.org/0000-0002-4003-5529

Financial support: None.

Conflict of interest: None.

Copyright(C) 2021 Korean Shoulder and Elbow Society.

This is an Open Access article distributed under the terms of the Creative Commons Attribution Non-Commercial License (http://creativecommons.org/licenses/by-nc/4.0/) which permits unrestricted non-commercial use, distribution, and reproduction in any medium, provided the original work is properly cited. 
outcomes: rotator cuff dysfunction, glenoid bone deformity, and preoperative stiffness. These three factors independently can influence the outcome of TSA but sometimes coexist and can influence each other $[9,10]$.

In terms of soft tissue balance, rotator cuff condition is the most important factor. Postoperative rotator cuff tear can cause instability, which can progress to glenoid loosening and failure. Preoperative rotator cuff tear, rotator cuff muscle atrophy (MA), and fatty infiltration (FI) are correlated with poor clinical results [1,9,11-13]. In terms of bony architecture, uncorrectable glenoid deformities (e.g., glenoid retroversion, posterior erosion, and humeral head subluxation) are negative factors related with poor clinical results after TSA. Joint stiffness is another negative factor commonly comorbid in TSA. The appropriate treatment of patients with GHOA and significant stiffness (limitation of motion) remains a controversial clinical dilemma $[1,14]$. Stiff shoulders can be associated with significant rotator cuff muscle dysfunction, even in the absence of a full-thickness rotator cuff tear. Advanced age and long-standing stiffness have been linked to increased FI and MA [10,13].

The goals of this study are (1) to describe the three conditions (rotator cuff dysfunction, glenoid bone deformity, and stiffness) in which RSA should be considered for treatment of GHOA, (2) to review the clinical and mechanical background of RSA and TSA, and (3) to review published clinical outcomes of RSA for treatment of GHOA.

\section{ROTATOR CUFF TEAR}

\section{Clinical Outcome of RSA in GHOA with Intact Cuff}

Recently, RSA has been performed for GHOA even in cases where the rotator cuff is preserved and has shown good results comparable with those of TSA. Wright et al. [14] compared TSA and RSA in patients 70 years and older with GHOA and an intact rotator cuff. There was no difference in patient-reported outcome measures, range of motion, American Shoulder and Elbow Surgeons (ASES) score, Western Ontario Osteoarthritis of the Shoulder index, or complication rate or revision surgery rate between the groups. All patients of the RSA group and $98 \%$ of the TSA group could achieve full or nearly full $\left(>135^{\circ}\right)$ forward elevation at a minimum of two years after the procedure [14]. Steen et al. [15] evaluated 24 consecutive GHOA patients who underwent RSA and matched them to 96 patients who underwent TSA. Postoperative ASES, Simple Shoulder Test score, and range of motion were similar between the groups. There was no significant difference in complication rate or revision surgery rate between groups. However, five TSA patients showed radiographic glenoid loosening, whereas no RSA patients did [15].

\section{Incidence of rotator cuff tear in GHOA}

The incidence of rotator cuff tear in the asymptomatic elderly population is high. Khoschnau et al. [16] evaluated prevalence of rotator cuff tears in a population with a mean age of 66 years who had never sought care for shoulder symptoms. Of the 106 individuals (212 shoulders), the prevalence of full-thickness cuff tear was $30 \%$ ( $21 \%$ of 212 shoulders). Another study investigated the clinical and ultrasonography results of shoulders from 420 asymptomatic volunteers aged between 50 and 79 years. Full-thickness tear of the rotator cuff was detected in 32 individuals (7.6\%). The prevalence increased with age as follows: 50 to 59 years, $2.1 \%$; 60 to 69 years, $5.7 \%$; and 70 to 79 years, $15 \%$ [17]. Minagawa et al. [18] evaluated 664 residents in one village who had undergone ultrasonography. The prevalence of rotator cuff tear in the general population was $22.1 \%$, which increased with age. Asymptomatic tear was twice as common as symptomatic tear. However, the incidence of rotator cuff tear in GHOA patients is controversial. Edwards et al. [13] described the results of TSA in 555 osteoarthritic shoulders, of which 42 (7.6\%) had a rotator cuff tear. In Iannotti and Norris's study [1], most $(n=115 ; 90 \%)$ of 128 shoulders had a structurally intact rotator cuff. Thirteen were found to have a full thickness tear, but only seven (5\% of 128) had a tear $>1 \mathrm{~cm}$. However, since patients with large rotator cuff tear might be excluded from TSA study, the incidence of rotator cuff tear could be underestimated.

\section{Significancy of rotator cuff in TSA}

In GHOA, rotator cuff conditions are variable in tear size, cuff thickness, MA, and FI. Sometimes, even without cuff tear, MA and FI can be severe, or rotator cuff tendon can be thin. Several classifications have been used to describe the condition of rotator cuff in terms of MA, FI, and tear size [19-21]. GHOA commonly is accompanied by degenerative changes in the rotator cuff [22]. However, none of the classifications adequately describe the degenerative degree of the rotator cuff.

The size of the rotator cuff tear before surgery should be considered carefully. A repairable tear of the supraspinatus tendon is not a contraindication to TSA. If partial tear or small rotator cuff tears are well repaired during TSA surgery, they have little influence on the results of shoulder arthroplasty [1]. Raval et al. [23] evaluated 36 patients with a mean age 79.2 years who underwent TSA and had GHOA with partial-thickness rotator cuff tears observed on MRI for a mean follow-up of 5.8 years. The study showed that presence of a partial cuff tear on preoperative MRI does not significantly affect function after anatomical TSA in the 
medium-term follow-up. However, a medium- to large-sized full thickness rotator cuff tear negatively influences the results of shoulder arthroplasty. Simone et al. [2] evaluated 33 patients who had rotator cuff repair with TSA for a mean follow-up of 4.7 years. Instability and glenoid loosening occurred in six patients with medium or large tear. Complications were noted in five patients, all with medium or large tear; four of these had symptomatic instability and one sustained a late peri-prosthetic fracture. Four patients required further surgery, three due to instability and one due to peri-prosthetic humeral fracture [2]. Coexistent tears of the rotator cuff prejudice the outcome of TSA by reducing active movement and strength and by predisposing to instability or subluxation of the replacement and loosening of the glenoid component (Fig. 1). Postoperative rotator cuff tears in TSA are not only related with decreased range of motion, but also instability or subluxation, which eventually lead to early glenoid loosening $[1,12,13,24]$.

Subscapularis tear after TSA is a common complication but cannot be diagnosed reliably by physical examination or radiographs. Although there is an opinion that subscapularis integrity does not correlate with pain or subjective patient outcome, inadequate healing of the subscapularis tendon can lead to postoperative pain, weakness, and instability [25-27]. Postoperative subscapularis tear could induce upward migration of the humeral head, anterosuperior subluxation, an eccentric contact pattern,
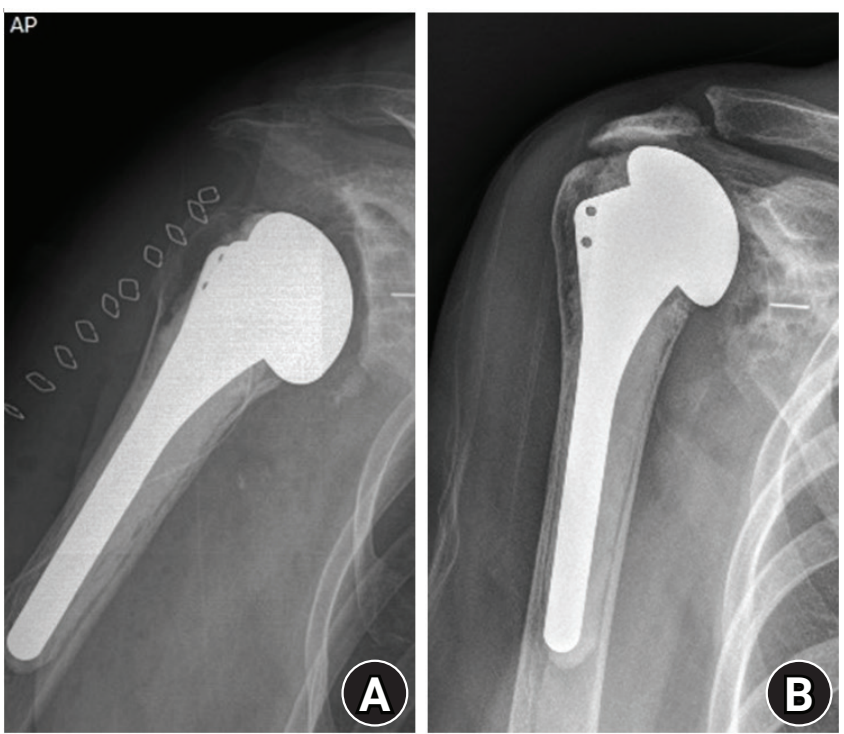

Fig. 1. (A) Immediate postoperative radiograph after total shoulder arthroplasty shows normal glenohumeral distance and contiguous scapulohumeral line. (B) In 3-year follow-up radiograph, superior migration (decreased acromiohumeral distance) and osteolysis around glenoid component are observed from the postoperative rotator cuff tear. and higher stress to the glenoid component [28].

The clinical significance of subscapularis repair is controversial in RSA. A prospective randomized trial by Engel et al. [29] concluded that subscapularis tendon repair in RSA improves the Constant score and internal rotation at 12 months after surgery. In medialized design RSA, the subscapularis has an important role in preventing dislocation [30]. Although subscapularis repair is safe and effective for RSA, it cannot offer additional clinical or functional benefit in patients treated with lateralized RSA [31]. Therefore, in GHOA with inadequate subscapularis condition where postoperative retear is expected, RSA could be considered.

In addition to rotator cuff tear, MA and FA should be considered preoperatively in TSA. In GHOA patients, FI and MA of the rotator cuff are major factors associated with clinical outcomes after TSA. Conversely, they are not significant in RSA. Puzzitiello et al. [12] concluded that rotator cuff muscle quality as assessed by MA and FI does not impact clinical outcomes following RSA with a lateralized glenosphere in patients with GHOA and an intact rotator cuff. Therefore, if progressed MA and FI is combined with GHOA, RSA could be a reasonable decision even with an intact rotator cuff $[2,10,12,13]$.

\section{GLENOID DEFORMITY (GLENOID BONE LOSS, POSTERIOR GLENOID WEAR, AND INCREASED RETROVER- SION)}

\section{Normal Anatomy of Glenoid}

Prosthetic design and surgical considerations related to glenoid anatomy are based on numerous studies focusing on glenoid height, width, inclination, and version [32]. The "normal" range of glenoid version varies anywhere from $2^{\circ}$ of anteversion to $8^{\circ}$ of retroversion in most studies [33-35]. Studies using three-dimensional measurement techniques on computed tomography images have reported native glenoid version of approximately $7^{\circ}$ [36,37]. However, arthritic shoulders generally have greater than $11^{\circ}$ of retroversion, which should be corrected during TSA [33, 38,39].

\section{Classification and Clinical Significance of Glenoid Deformity}

Walch et al. [40,41] devised a classification system for glenoid morphology that is based on the architecture and patterns of posterior wear in GHOA [32]. In type B2 glenoids, posterior humeral head subluxation and posterior glenoid wear can increase glenoid retroversion to values above $10^{\circ}$. Type $\mathrm{C}$ glenoids with evidence of dysplasia can show glenoid retroversion above $25^{\circ}$. 
Failure to replicate and restore characteristics of the normal glenoid articular surface can lead to early loosening. This can be particularly difficult in patients with biconcave glenoids and associated posterior humeral head instability. Failure to restore neutral glenoid version can increase the shear load across the glenoid. This subtype has problems in soft tissue balancing and is associated with a high rate of revision surgery because of glenoid loosening and instability [11,32,41,42].

Posterior wear and increased retroversion in glenohumeral arthritis often is associated with static posterior subluxation of the humeral head. Static posterior subluxation could be reversed by TSA using corrective glenoid reaming and soft tissue release [43]. However, if static posterior subluxation persists after TSA, postoperative subluxation can lead to eccentric loading of the glenoid component and accelerated loosening and wear [11,32,41,43].

Variable methods of correcting version and increasing stability of the glenoid component have been used intraoperatively. Asymmetric glenoid reaming, posterior glenoid bone grafting, and use of specialized glenoid implants are included. The choice among these options is based on the ability to assess accurately both glenoid version and the desired amount of correction intraoperatively. However, this often is limited by obscured bony landmarks and deficient bone stock. Even with preoperative three-dimensional imaging, implantation of a glenoid component to within $10^{\circ}$ of a desired version is technically difficult even for an experienced shoulder surgeon in cases of severe retroversion [44].

\section{Bone Grafting in TSA and RSA}

Bone graft could be used in TSA in patients with osteoarthritis combined with increased retroversion or biconcavity. Bone grafting with internal fixation is a reconstructive technique with mixed clinical results. It has been used in limited cases of large segmental bone deficiencies or in cases of severe posterior wear causing severe component loosening. However, a very high rate of complications after bone graft such as bone resorption, nonunion, and early loosening were found. Because of the high complication rate of posterior bone graft with an anatomic prosthesis, Walch et al. [41] recommend RSA instead of TSA with neoglenoid retroversion $[11,32,44]$. However, the bone healing rate is very high and predictable in RSA [45].

\section{DIFFERENCES IN BONE GRAFT BE- TWEEN TSA AND RSA}

RSA has some advantages compared to TSA. (1) Stable fixation: variable angle locking screw fixation creates a more stable con- struct and reduces baseplate micromotion. However, in TSA, cement-type fixation for glenoid components has been used commonly and can interfere with bone union. Bone grafting with internal fixation is a technically demanding procedure in TSA. The period of motion restriction can be prolonged, and the success rate is not high [32]. (2) Diminished force to graft: unequal radii of curvature between the humeral and glenoid components of TSA permit translation movement, which produces shearing force between the glenoid component and bone graft. However, a reverse prosthesis, designed with equal radii of curvature, can tolerate a joint-reaction force vector. Increased constraint secondary to the deeper and greater conformity of the concavity of the humeral articular surface prevents glenohumeral translation while providing sufficient stability $[8,46]$. (3) As the burden of restoring the joint line and soft tissue balance is lower in RSA than TSA, glenoid bone graft could be thick enough to achieve firm fixation. (4) It is easier to correct glenoid version in RSA than TSA as an asymmetric bone graft is possible [47-49].

\section{STIFFNESS}

Joint stiffness can cause difficulties in any surgery performed on the shoulder joint. In TSA, preoperative stiffness corresponds to a major risk of poor clinical results, and recovery of range of motion is difficult even after surgery. Stiffness is the most common cause of failure in TSA and postoperative stiffness has been considered a type of failure [9]. Joint stiffness can be confirmed by a decrease in passive range of motion [1].

The difficulties encountered during TSA procedure in a stiff shoulder joint are as follows. (1) Joint stiffness deteriorates the operation field exposure for the glenoid procedure, which is one of the reasons for difficult operation. (2) If the glenoid is not exposed sufficiently, problems can occur during glenoid preparation and glenoid component positioning. This condition could increase the risk of glenoid malposition, which leads to early loosening of the glenoid implant [50]. (3) Nerve injury is very rare in shoulder arthroplasty. However, in shoulders with stiffness, the risk of nerve injury could increase as excessive soft tissue traction or soft tissue release often is required [51-53]. (4) It often is difficult to repair the rotator cuff after implantation. The rotator cuff of a shoulder with longstanding stiffness could be irreparable after implantation or have short tendon excursion. In this condition, repair of the subscapularis can be incomplete or not possible, which could increase the extent of future subscapularis tear [3,9].

In RSA on a stiff shoulder, there are many difficulties. As the glenoid component of RSA is larger than that of TSA, a larger ex- 
posure is required in RSA. In addition, the risk of fracture could increase during the reduction step of the procedure. Due to the convexity of the glenoid component, reduction requires sufficient traction. During this step, greater tuberosity fracture could occur. As the humerus is retracted posteriorly using a traction tool to expose the glenoid in the stiff shoulder joint, bone erosion or fracture by the traction tool can occur in the humeral head [54].

Despite the above difficulties, RSA is preferred for GHOA with stiffness because the results of TSA are inferior. Though there is no study comparing TSA and RSA in GHOA with stiffness, RSA could result in potentially good results even in stiff shoulders [54]. There are several technical advantages of RSA in GHOA with stiffness. (1) Freedom from rotator cuff preservation: in TSA, the rotator cuff tendon should be preserved and repaired at the last stage of surgery. In addition, MA and FI are important [10]. However, in RSA, the tendons of supraspinatus and infraspinatus can be removed, and MA and FI are not critical to clinical outcomes [12]. Moreover, superior rotator cuff removal could improve surgical field exposure and allows soft tissue release [12,31]. (2) Greater capacity of humeral bone cutting: in RSA compared to TSA, greater humeral head cutting is possible beyond the rotator cuff attachment. Increased humeral bone cutting improves the surgical field exposure and stiffness. (3) Freedom from subscapularis repair: as described in the previous paragraph, lateralized implantation of RSA does not require subscapularis repair. This is helpful to reduce soft tissue tension during operation and to prevent postoperative stiffness $[31,55]$.

\section{CONCLUSIONS}

The indications for RSA gradually are expanding. For GHOA with intact rotator cuff, TSA is the gold standard treatment. However, RSA could be adopted. It is reasonable that RSA is selected preferentially for treatment of GHOA in which specific conditions are combined, such as rotator cuff degeneration (greater than $1 \mathrm{~cm}$ tear, advanced MA or FI, subscapularis insufficiency), glenoid bone deformity (glenoid bone loss or retroversion needing bone graft), and stiffness.

\section{ORCID}

$\begin{array}{ll}\text { Young-Hoon Jo } & \text { https://orcid.org/0000-0002-4299-2496 } \\ \text { Dong-Hong Kim } & \text { https://orcid.org/0000-0002-9088-7551 } \\ \text { Bong Gun Lee } & \text { https://orcid.org/0000-0002-4003-5529 }\end{array}$

\section{REFERENCES}

1. Iannotti JP, Norris TR. Influence of preoperative factors on outcome of shoulder arthroplasty for glenohumeral osteoarthritis. J Bone Joint Surg Am 2003;85:251-8.

2. Simone JP, Streubel PH, Sperling JW, Schleck CD, Cofield RH, Athwal GS. Anatomical total shoulder replacement with rotator cuff repair for osteoarthritis of the shoulder. Bone Joint J 2014; 96:224-8.

3. Neer CS 2nd, Watson KC, Stanton FJ. Recent experience in total shoulder replacement. J Bone Joint Surg Am 1982;64:319-37.

4. Hyun YS, Huri G, Garbis NG, McFarland EG. Uncommon indications for reverse total shoulder arthroplasty. Clin Orthop Surg 2013;5:243-55.

5. Berliner JL, Regalado-Magdos A, Ma CB, Feeley BT. Biomechanics of reverse total shoulder arthroplasty. J Shoulder Elbow Surg 2015;24:150-60.

6. Lorenzetti AJ, Stone GP, Simon P, Frankle MA. Biomechanics of reverse shoulder arthroplasty: current concepts. Instr Course Lect 2016;65:127-43.

7. Wall B, Nové-Josserand L, O'Connor DP, Edwards TB, Walch G. Reverse total shoulder arthroplasty: a review of results according to etiology. J Bone Joint Surg Am 2007;89:1476-85.

8. Friedman RJ. Biomechanics of total shoulder arthroplasty: a preoperative and postoperative analysis. Semin Arthroplasty $1995 ; 6: 222-32$

9. Hasan SS, Leith JM, Campbell B, Kapil R, Smith KL, Matsen FA 3rd. Characteristics of unsatisfactory shoulder arthroplasties. J Shoulder Elbow Surg 2002;11:431-41.

10. Moverman MA, Puzzitiello RN, Menendez ME, et al. Rotator cuff fatty infiltration and muscle atrophy: relation to glenoid deformity in primary glenohumeral osteoarthritis. J Shoulder Elbow Surg 2021 Aug 11 [Epub]. https://doi.org/10.1016/j.jse. 2021.07.007.

11. Hussey MM, Steen BM, Cusick MC, et al. The effects of glenoid wear patterns on patients with osteoarthritis in total shoulder arthroplasty: an assessment of outcomes and value. J Shoulder Elbow Surg 2015;24:682-90.

12. Puzzitiello RN, Moverman MA, Menendez ME, Hart PA, Kirsch J, Jawa A. Rotator cuff fatty infiltration and muscle atrophy do not impact clinical outcomes after reverse total shoulder arthroplasty for glenohumeral osteoarthritis with intact rotator cuff. J Shoulder Elbow Surg 2021;30:2506-13.

13. Edwards TB, Boulahia A, Kempf JF, Boileau P, Nemoz C, Walch G. The influence of rotator cuff disease on the results of shoulder arthroplasty for primary osteoarthritis: results of a multicenter study. J Bone Joint Surg Am 2002;84:2240-8. 
14. Wright MA, Keener JD, Chamberlain AM. Comparison of clinical outcomes after anatomic total shoulder arthroplasty and reverse shoulder arthroplasty in patients 70 years and older with glenohumeral osteoarthritis and an intact rotator cuff. J Am Acad Orthop Surg 2020;28:e222-9.

15. Steen BM, Cabezas AF, Santoni BG, et al. Outcome and value of reverse shoulder arthroplasty for treatment of glenohumeral osteoarthritis: a matched cohort. J Shoulder Elbow Surg 2015;24: 1433-41.

16. Khoschnau S, Milosavjevic J, Sahlstedt B, Rylance R, Rahme H, Kadum B. High prevalence of rotator cuff tears in a population who never sought for shoulder problems: a clinical, ultrasonographic and radiographic screening study. Eur J Orthop Surg Traumatol 2020;30:457-63.

17. Moosmayer S, Smith HJ, Tariq R, Larmo A. Prevalence and characteristics of asymptomatic tears of the rotator cuff: an ultrasonographic and clinical study. J Bone Joint Surg Br 2009; 91:196-200.

18. Minagawa H, Yamamoto N, Abe H, et al. Prevalence of symptomatic and asymptomatic rotator cuff tears in the general population: from mass-screening in one village. J Orthop 2013;10: 8-12.

19. Warner JJ, Higgins L, Parsons IM 4th, Dowdy P. Diagnosis and treatment of anterosuperior rotator cuff tears. J Shoulder Elbow Surg 2001;10:37-46.

20. Goutallier D, Postel JM, Gleyze P, Leguilloux P, Van Driessche S. Influence of cuff muscle fatty degeneration on anatomic and functional outcomes after simple suture of full-thickness tears. J Shoulder Elbow Surg 2003;12:550-4.

21. Davidson J, Burkhart SS. The geometric classification of rotator cuff tears: a system linking tear pattern to treatment and prognosis. Arthroscopy 2010;26:417-24.

22. Ibrahim M, Kartus JT, Steigen SE, Olsen R, Meknas K. More tendon degeneration in patients with shoulder osteoarthritis. Knee Surg Sports Traumatol Arthrosc 2019;27:267-75.

23. Raval P, Gibbs VN, Pandey R. Preoperative partial-thickness rotator cuff tears do not compromise anatomic total shoulder replacement outcomes: medium-term follow-up. J Shoulder Elbow Surg 2021;30:871-6.

24. Godenèche A, Boileau P, Favard L, et al. Prosthetic replacement in the treatment of osteoarthritis of the shoulder: early results of 268 cases. J Shoulder Elbow Surg 2002;11:11-8.

25. Jackson JD, Cil A, Smith J, Steinmann SP. Integrity and function of the subscapularis after total shoulder arthroplasty. J Shoulder Elbow Surg 2010;19:1085-90.

26. Qureshi S, Hsiao A, Klug RA, Lee E, Braman J, Flatow EL. Subscapularis function after total shoulder replacement: results with lesser tuberosity osteotomy. J Shoulder Elbow Surg 2008; 17:68-72.

27. Shields E, Ho A, Wiater JM. Management of the subscapularis tendon during total shoulder arthroplasty. J Shoulder Elbow Surg 2017;26:723-31.

28. Terrier A, Larrea X, Malfroy Camine V, Pioletti DP, Farron A. Importance of the subscapularis muscle after total shoulder arthroplasty. Clin Biomech (Bristol, Avon) 2013;28:146-50.

29. Engel NM, Holschen M, Schorn D, Witt KA, Steinbeck J. Results after primary reverse shoulder arthroplasty with and without subscapularis repair: a prospective-randomized trial. Arch Orthop Trauma Surg 2021 Jul 8 [Epub]. https://doi.org/10. 1007/s00402-021-04024-6.

30. Matthewson G, Kooner S, Kwapisz A, Leiter J, Old J, MacDonald $\mathrm{P}$. The effect of subscapularis repair on dislocation rates in reverse shoulder arthroplasty: a meta-analysis and systematic review. J Shoulder Elbow Surg 2019;28:989-97.

31. Roberson TA, Shanley E, Griscom JT, et al. Subscapularis repair is unnecessary after lateralized reverse shoulder arthroplasty. JB JS Open Access 2018;3:e0056.

32. Hsu JE, Ricchetti ET, Huffman GR, Iannotti JP, Glaser DL. Addressing glenoid bone deficiency and asymmetric posterior erosion in shoulder arthroplasty. J Shoulder Elbow Surg 2013; 22:1298-308.

33. Friedman RJ, Hawthorne KB, Genez BM. The use of computerized tomography in the measurement of glenoid version. J Bone Joint Surg Am 1992;74:1032-7.

34. Churchill RS, Brems JJ, Kotschi H. Glenoid size, inclination, and version: an anatomic study. J Shoulder Elbow Surg 2001;10:32732.

35. Nyffeler RW, Jost B, Pfirrmann CW, Gerber C. Measurement of glenoid version: conventional radiographs versus computed tomography scans. J Shoulder Elbow Surg 2003;12:493-6.

36. Ganapathi A, McCarron JA, Chen X, Iannotti JP. Predicting normal glenoid version from the pathologic scapula: a comparison of 4 methods in 2- and 3-dimensional models. J Shoulder Elbow Surg 2011;20:234-44.

37. Hendel MD, Bryan JA, Barsoum WK, et al. Comparison of patient-specific instruments with standard surgical instruments in determining glenoid component position: a randomized prospective clinical trial. J Bone Joint Surg Am 2012;94:2167-75.

38. Scalise JJ, Codsi MJ, Bryan J, Iannotti JP. The three-dimensional glenoid vault model can estimate normal glenoid version in osteoarthritis. J Shoulder Elbow Surg 2008;17:487-91.

39. Bokor DJ, O'Sullivan MD, Hazan GJ. Variability of measurement of glenoid version on computed tomography scan. J Shoulder Elbow Surg 1999;8:595-8. 
40. Walch G, Badet R, Boulahia A, Khoury A. Morphologic study of the glenoid in primary glenohumeral osteoarthritis. J Arthroplasty 1999;14:756-60.

41. Walch G, Moraga C, Young A, Castellanos-Rosas J. Results of anatomic nonconstrained prosthesis in primary osteoarthritis with biconcave glenoid. J Shoulder Elbow Surg 2012;21:152633.

42. Nyffeler RW, Sheikh R, Atkinson TS, Jacob HA, Favre P, Gerber C. Effects of glenoid component version on humeral head displacement and joint reaction forces: an experimental study. J Shoulder Elbow Surg 2006;15:625-9.

43. Gerber C, Costouros JG, Sukthankar A, Fucentese SF. Static posterior humeral head subluxation and total shoulder arthroplasty. J Shoulder Elbow Surg 2009;18:505-10.

44. Iannotti JP, Greeson C, Downing D, Sabesan V, Bryan JA. Effect of glenoid deformity on glenoid component placement in primary shoulder arthroplasty. J Shoulder Elbow Surg 2012;21:4855.

45. Boileau P, Moineau G, Roussanne Y, O'Shea K. Bony increased offset-reversed shoulder arthroplasty (BIO-RSA). JBJS Essent Surg Tech 2017;7:e37.

46. Matsen FA 3rd, Boileau P, Walch G, Gerber C, Bicknell RT. The reverse total shoulder arthroplasty. J Bone Joint Surg Am 2007; 89:660-7.

47. Dimock R, Fathi Elabd M, Imam M, Middleton M, Godenèche A, Narvani AA. Bony increased-offset reverse shoulder arthroplasty: a meta-analysis of the available evidence. Shoulder Elbow 2021;13:18-27.
48. Choi WS, Lee KH, Park JS, Lee BG. Bilateral bony increased-offset reverse shoulder arthroplasty in rheumatoid arthritis shoulder with severe glenoid bone defect: a case report. Acta Orthop Traumatol Turc 2017;51:262-5.

49. Boileau P, Moineau G, Roussanne Y, O'Shea K. Bony increased-offset reversed shoulder arthroplasty: minimizing scapular impingement while maximizing glenoid fixation. Clin Orthop Relat Res 2011;469:2558-67.

50. Nové-Josserand L, Clavert P. Glenoid exposure in total shoulder arthroplasty. Orthop Traumatol Surg Res 2018;104:S129-35.

51. Florczynski M, Paul R, Leroux T, Baltzer H. Prevention and treatment of nerve injuries in shoulder arthroplasty. J Bone Joint Surg Am 2021;103:935-46.

52. LiBrizzi CL, Rojas J, Joseph J, Bitzer A, McFarland EG. Incidence of clinically evident isolated axillary nerve injury in 869 primary anatomic and reverse total shoulder arthroplasties without routine identification of the axillary nerve. JSES Open Access 2019;3:48-53.

53. Parisien RL, Yi PH, Hou L, Li X, Jawa A. The risk of nerve injury during anatomical and reverse total shoulder arthroplasty: an intraoperative neuromonitoring study. J Shoulder Elbow Surg 2016;25:1122-7.

54. Carofino B, Routman H, Roche C. The influence of preoperative external rotation weakness or stiffness on reverse total shoulder arthroplasty. JSES Int 2020;4:382-7.

55. Jawa A, Colliton EM. Role of subscapularis tendon repair in reverse total shoulder arthroplasty. J Am Acad Orthop Surg 2021; 29:604-8. 